\title{
A DEM MODEL TO PREDICT AND CORRECT SPREADER SHEAR-INDUCED PART DEFORMATION IN BINDER JET ADDITIVE MANUFACTURING
}

\author{
PRATHAMESH S. DESAI ${ }^{1}$ AND C. FRED HIGGS III ${ }^{2}$ \\ ${ }^{1}$ W. M. Rice University \\ 6100 Main St, Houston, TX 77005 \\ pratnsai@gmail.com and https://sites.google.com/site/pratnsaiweb/ \\ ${ }^{2}$ W. M. Rice University \\ 6100 Main St, Houston, TX 77005 \\ higgs@ rice.edu and https://higgslab.org/
}

Key words: Additive Manufacturing (AM), powder rheometry, virtual 3D printed cuboid,Discrete Element Method (DEM), shear-induced part deformation

\begin{abstract}
Powder bed additive manufacturing (AM) is comprised of two repetitive steps: spreading of powder and selective fusing or binding the spread layer. Powder-bed AM can be sub-categorized as fusion-based where electron beams or laser beams are used to fuse the spread powder layer and binder-based where a liquid binder is used to bind the spread layer at areas specified by the governing CAD model. The latter process, commonly referred to as binder jet additive manufacturing (BJAM), outperforms fusion-based methods with respect to cost, build time, and material suitability; however, the parts are prone to shear-induced deformation during the powder spreading stage. Unlike fusionbased AM, the strength of BJAM parts is not fully developed until sintering and infiltration during postprocessing. This results in BJAM parts being more susceptible to deformation or even breakage due to the shearing action of the spreader. This shear-induced deformation can affect the precision and thereby performance of 3D printed parts. The binding step in BJAM is a complex function of binder viscosity, density, droplet size, impact speed, and drying time. The spreading step is a complex function of spreader speed and spreader shape, topography of spread and bound layer, and the rheology of the AM powder. This study presents a first-order model to simulate BJAM using a weak concrete-like, non-local, multilayer bonded DEM model. The DEM model has been parallelized using the massive parallelism offered by GPUs. An industry-grade metal powder is used to print physical cuboids at varying spreader speeds. The model is qualitatively verified against experiments on a real 3D printer. The model can be used to provide layer-wise spreading process control to minimize spreader shear-induced deformations.
\end{abstract}




\section{Nomenclature}

\begin{tabular}{||ll||}
\hline Symbol & Meaning \\
\hline \hline$K$ & Stiffness of the spring in a spring-dashpot system \\
$K_{\text {bond }}$ & Stiffness of the mathematical bond between particles \\
$\gamma$ & Bond stiffness factor \\
$\beta$ & Damping of dashpot in a spring-dashpot system \\
$n, t$ & Subscripts: normal and tangential directions respectively \\
$\varepsilon$ & Coefficient of restitution \\
$p p, p s, p b$ & Subscripts: Collisions occurring between a particle (p) and another particle (p), spreader (s) or build box (b) respectively \\
$m$ & Particle mass \\
$V$ & Particle speed \\
$\Delta$ & Overlap of a particle with another particle or geometry \\
$\phi$ & Diameter of a spherical particle \\
$\mu$ & Coefficient of sliding friction \\
$\sigma_{b o n d}$ & Bond strength in newtons \\
$\frac{C}{e_{t}}$ & Position vector of the center of a spherical particle \\
$\lambda$ & Unit vector along the tangential direction \\
$F$ & Bond strength factor \\
\hline
\end{tabular}

\section{INTRODUCTION}

The additive manufacturing process comprises three repetitive steps: delivery of the powder from a hopper, spreading the powder in a thin layer, and selective fusing or binding the spread layer $[1,2,3,4]$. Powder-bed AM can be sub-categorized into fusion-based where electron beams or laser beams are used to fuse the spread powder layer and binder-based where a liquid binder is used to bind the spread layer at areas specified by additive manufacturing part deformation problem not held as tightly together when compared to the to deformation or even breakage dute to the sheari
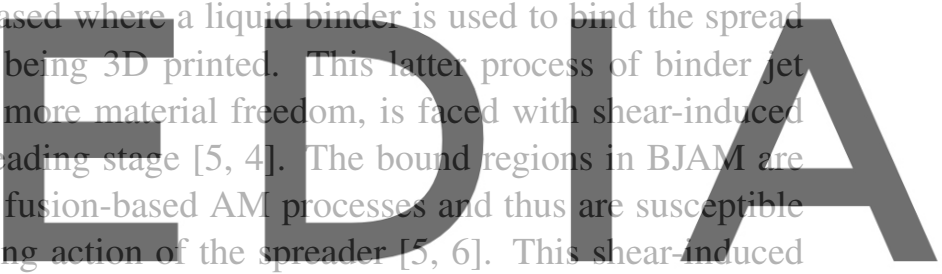

deformation can affect the precision and, thereby, the performance of 3D printed parts. The binding step

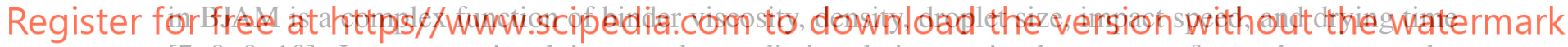
$[7,8,9,10]$. In a system involving powder media in relative motion between surfaces, the topography and micro-scale surface features in concert with the powder rheology are a function of the performance of the loose and bound powder $[6,11,8]$. Thus, there is a need to understand better the powder rheology and powder-boundary surface effects, which control the spread layer defects of porosity, roughness, and part deformation.

For the spreading step in powder-bed 3D printing, the Holy Grail has CAD-specific spreading control software that can tell the 3D printer the process parameters to use to spread any powder to produce high quality and defect-free parts. However, such a model will have to account for many attributes related to powder dynamics like particle shape, particle size distribution, particle adhesion and cohesion, particle roughness, magnetic or electrostatic behavior of the particles, etc. This model must also accurately treat the solid surfaces (e.g., spreader geometry and spreading substrate) and simulate realistic particle counts (i.e., 10-100 million). It is near impossible to develop such an all-inclusive physics-based model that is computationally efficient.

The jetting pattern encountered in BJAM, schematically shown in Fig. 1, is quite complex. The jetting action is a highly temporal process and the impact of binder droplets on the spread layer can significantly 
$S_{L}=$ Soak Length i.e., the size of voxel or primitive.

$J_{S}=$ Jet Spacing.

Point $\quad \underline{\text { Line }} \quad$ Rectangle

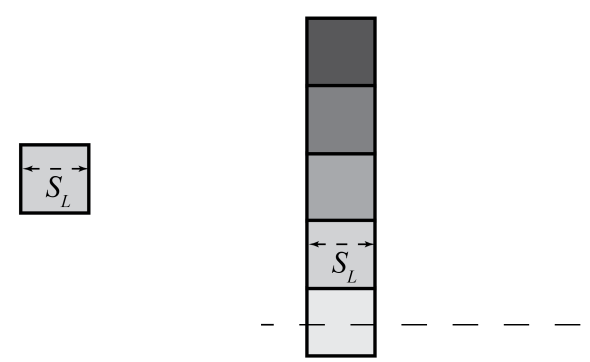

1

।

$\underline{\text { Jets' trigger times } T \text { 's }}$
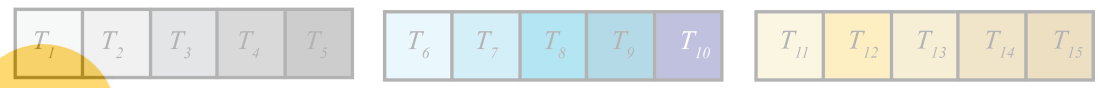

Figure 1. Typical jetting action in a commercial BJAM 3D printer. Left When the printer tries to print a point or primitive or voxel, a single jet is triggered once. Center When the printer tries to print a line (i.e., a combination of points), based on the orientation of the line, a single jet may be triggered multiple times (shown) or multiple jets may be triggered simultaneously (not shown). Right When the printer tries to print a rectangle or any CAD-specific cross-section, multiple jets are triggered over multiple times to achieve the desired binding.

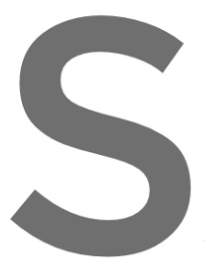

alter the layer roughness and porosity [10]. Ge et al. [12], and Zhang et al. 13) have experimentally
studied the strength of 3D printed agglomerates using BJAM. The findings from these studies yould
serve as a useful data set for validatimg multi-layer BJAM models.
Few researchers, including the authors of the present study, have tried to develop high-fidelity spreading models using discrete element method (DEM) $[14,15,16,17]$. However, all these models look at the

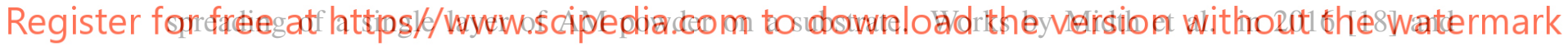
Steuben et al. in 2016 [19] are the only ones that have simulated 3D printing of an entire virtual object by accounting for spreading and fusion steps. Mindt et al. have used only a few particles and simulated only 3 spread and fused layers [18]. Steuben et al. have used first-order powder deposition models using the rainfall approach, spreading using a compressive rake, and powder fusion with no phase change [19].

To the best of our knowledge, this is the first study that models the multi-layer 3D printing process encountered in binder jet additive manufacturing (BJAM) and provides guidance on the control of the spreading process parameters(viz., spreader speed) to mitigate shear-induced part deformation. The methodology for the study is described in Section 2, preliminary experimental and modeling results are mentioned in Section 4, and finally conclusions are given in Section 4.

\section{METHODOLOGY}

\subsection{BJAM experiments on a commercial 3D printer}

In this work, a simple cuboid (refer to Fig. 2) was 3D printed at various spreader speeds and part orientation. Such a Design of Experiments (DoE) with only five variations per input variable can result 


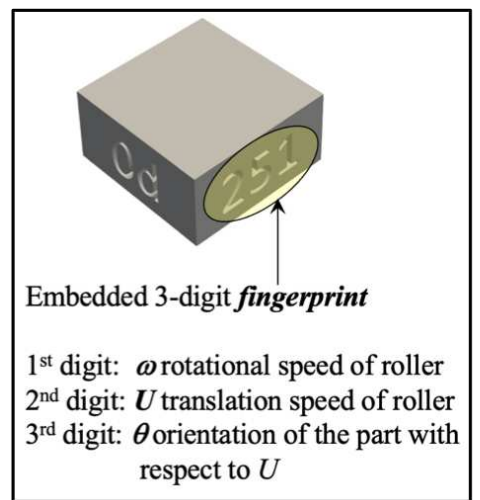

(a)

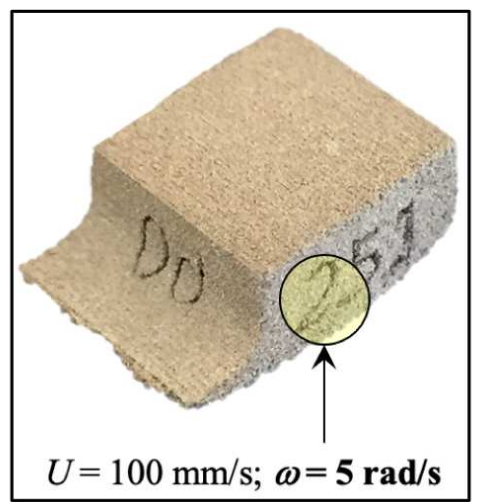

(b-left)

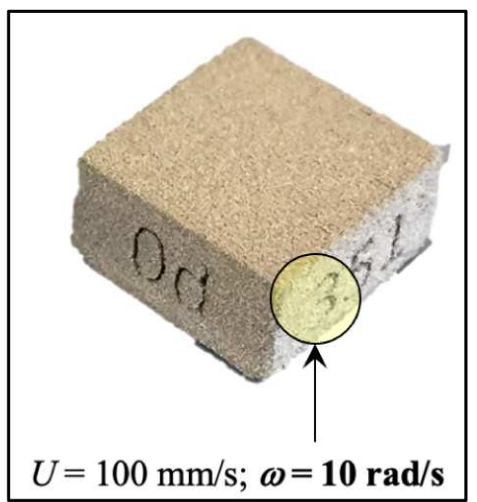

(b-right)

Figure 2: 3D printed cuboids by ExOne Innovent 3D printer- (a) CAD of the cuboid to be 3D printed. The CAD has an embedded 3-digit fingerprint. The size of the cuboid is $10 \mathrm{~mm}$ by $10 \mathrm{~mm}$ by $5 \mathrm{~mm}$. (b-left) Excessive shear deformation at certain spreader speed. (b-right) defect-free cuboid when optimal spreader speed was used, all other printing parameters were constant for the 3D printed cuboids in (b).

in hundreds of cubes, and keeping track of almost identical-looking cubes can easily get chaotic. A novel way, made possible only due to 3D printing, was used to generate a 3-digit fingerprint of the cube. This fingerprint that was a vector for numbered values $(\omega, U, \theta)$ was 3D printed on one side of the cube with

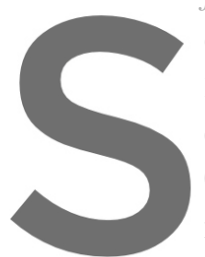
details of the individual par $20\}, U$ had values in $\mathrm{min} /$ examples, if $\omega$ is $5 \mathrm{rad} / \mathrm{s}$, $0^{\circ}$ ), then the fingerprint of th identity can be found on
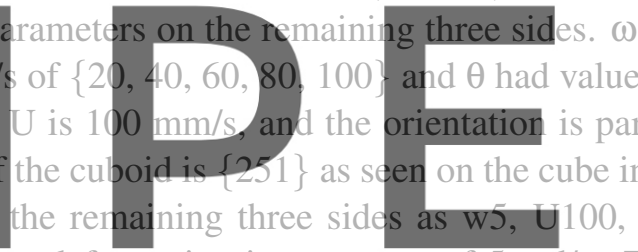

spreader shear-induced part deformation is seen at $\omega$ of $5 \mathrm{rad} / \mathrm{s}$.
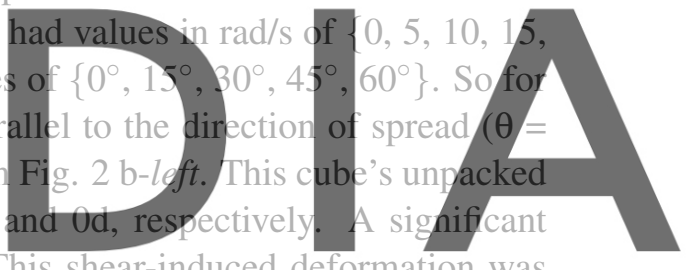
Register for free at htthe rotational speed of the spreader was increased to $10 \mathrm{rad} / \mathrm{s}$ (refer to Fig, $2 \mathrm{~b}-\mathrm{right}$. not visible to a camera. These shear-induced defects can not be corrected by imaging-based techniques such as the one developed by Scime et al. [20]. Only physics-based models of the entire BJAMI process may provide deeper insights into these defects. The modeling technique used in this study is described in the next subsection.

\subsection{BJAM physics-based computational modeling}

\subsubsection{Non-local bonded particle DEM model}

Bonded particle model making use of parallel bonds has been proposed by Cundall and Potyondy [21]. This model captures the heterogeneity of rocks by forming mathematical bonds, parallel or point, between overlapping particles. This model does not allow boding between particles that do not overlap but are in the vicinity of each other. Such types of bonds which are more intuitive and realistic (like cement paste biding aggregates in concrete) have been employed in the current study [22]. The schematic of the primitive modeled using non-local bonded DEM model is shown in Fig. 3. In this study the authors assume that all the primitives are formed simultaneously, are spherical in shape, and the spread layer is 


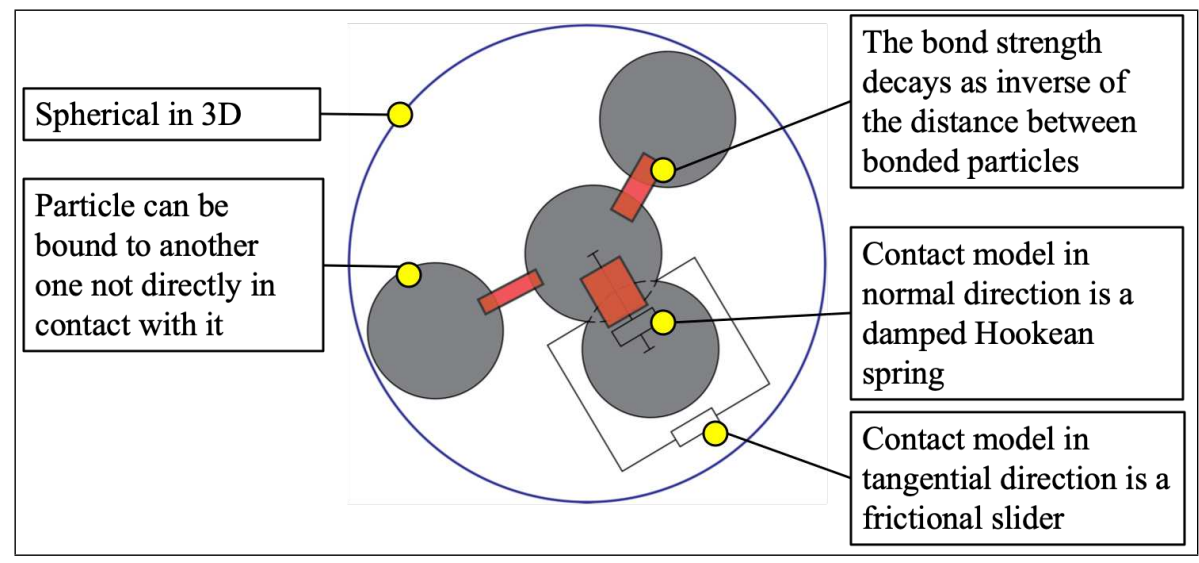

Figure 3: Primitive modeled using non-local bonded particle DEM

not deformed during the primitive formation. These first-order simplifying assumptions help to speed up the simulations.

Cement paste like mathematical bonds, shown as red rectangles in Fig. 3, are formed between uniform sized spherical, uncrushable particles. These bonds have a stiffness, referred to as $K_{\text {bond }}$, which is chosen to be a fraction of $K_{n}$ (Eq. 1). Here is $\gamma$ is referred to as bond stiffness factor.
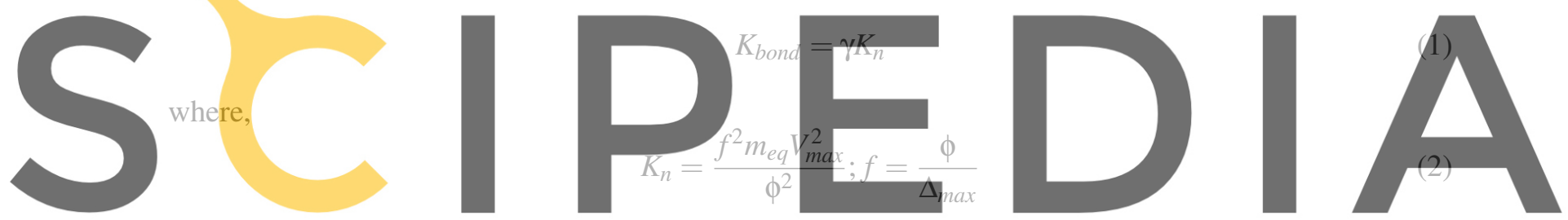

Here $m_{e q}$ stands for the equivalent mass of colliding particles, having diameter $\phi$ and constant coefficient

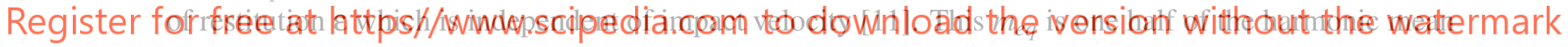
of the individual masses. $V_{\max }$ and $\Delta_{\max }$ are the estimated maximum speed and inter-particle penetration for the simulation at hand. These values are usually guessed. A technique to calibrate these values for additive manufacturing powders is described in [11].

The bonds have strengths $\sigma_{b o n d, i j}$ which decay as the distance between particles increases:

$$
\sigma_{b o n d, i j}=\frac{\phi}{\left\|\underline{C_{i}}-\underline{C_{j}}\right\|} \sigma_{b o n d, \max }
$$

Where $C_{i}$ and $C_{j}$ are the position vectors of the centers of particles $i$ and $j$ respectively and $\sigma_{b o n d, \max }$ is the absolute maximum bond strength. This absolute maximum bond strength is defined as the maximum force which the bond can resist before breaking:

$$
\sigma_{\text {bond, } \max }=\lambda K_{\text {bond }} \Delta_{\max }
$$

Here $\lambda$ is referred to as the bond strength factor. A slider is also present in the shear direction. It limits the maximum frictional force in this direction, the value of which is equal to the product of sliding 
friction coefficient and normal reaction force $F_{n}$ (given by Eq. 7). It is assumed that all the interactions cause particles to slide thereby nullifying the tangential damped Hookean spring. In other words, only the slider acts in the shear direction. Therefore, the forces along the normal $\left(F_{n}\right)$ and tangential $\left(\underline{F_{t}}\right)$ directions experienced by a colliding particle with an overlap of $\Delta$ with other particles or geometries, relative approach speed of $\dot{\Delta}$ and unit vector $e_{t}$ in shear direction as can be represented as:

$$
\begin{gathered}
\underline{F_{n}}= \begin{cases}K_{n} \underline{\Delta_{n}}-\beta_{n} \dot{\dot{\Delta}}_{n}+K_{b o n d} \underline{\Delta_{b o n d, n},} & \text { if } K_{b o n d}\left|\underline{\Delta_{b o n d}}\right|<\sigma_{b o n d} \text { (intact bond). } \\
K_{n} \underline{\Delta_{n}}-\beta_{n} \underline{\dot{\Delta}_{n}}, & \text { for (broken bond). }\end{cases} \\
\underline{F_{t}}= \begin{cases}-\mu\left|\underline{F_{n}}\right| \underline{e_{t}}+K_{b o n d} \underline{\Delta_{b o n d, t}}, & \text { if } K_{\text {bond }}\left|\underline{\Delta_{b o n d}}\right|<\sigma_{b o n d} \text { (intact bond). } \\
-\mu\left|\underline{F_{n}}\right| \underline{e_{t}}, & \text { for (broken bond). }\end{cases}
\end{gathered}
$$

The damping coefficient for the DEM particles is given by:

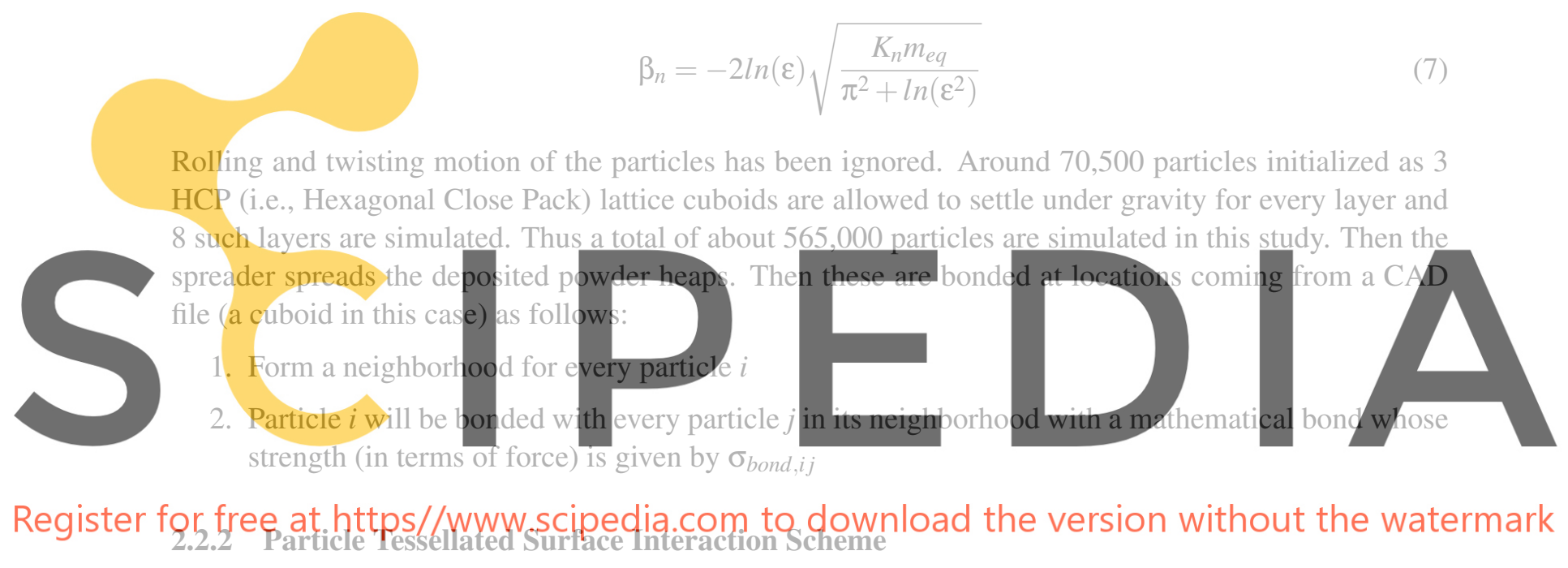

In the DEM modeling of the AM spreading process, particle and solid surface contact detection is important to efficiently and accurately capture the interaction between the AM powder and solid surfaces. Several methods can be used to represent these solid geometries. One way to represent solid geometries is to cover the solid surfaces (spreader and previously 3D-printed bottom surface) with spheres and moving these based on the prescribed motion of the solid surfaces [14]. This simplifies the calculation of particle-solid surface contact as these contacts are now simply particle-particle contact. The disadvantage of this treatment of solid surfaces is not able to capture the solid geometry accurately. In this study, we convert the solid geometries (viz., spreader, build platform, and printer box) into tessellated surfaces using SolidWorks' in-built stl generator [23] and particle-solid contact is modeled as particle-tesellated solid contact. The algorithm is inspired by the works of Passerello [24] and Hu et al. [25]. A complete description of the implemented algorithm can be found here [22]. 
Table 1: Contact interaction parameters used in DEM simulations

\begin{tabular}{||cc||}
\hline Parameter & Value \\
\hline \hline Density of grains $\left(\mathrm{kg} / \mathrm{m}^{3}\right)$ & 4430 \\
Grain diameter $(\mu \mathrm{m})$ & 500 \\
Bond influence region & $2 *$ grain diameter \\
$\mu_{p p}$ & 0.22 \\
$\mu_{p s}$ & 0.12 \\
$\mu_{p b}$ & 0.25 \\
$V_{\max }(\mathrm{mm} / \mathrm{s})$ & 80 \\
$\Delta_{\max }$ & $0.5 \%$ grain diameter \\
$\varepsilon$ & 0.8 \\
$\lambda$ & 10 \\
$\gamma$ & 0.8 \\
\hline
\end{tabular}

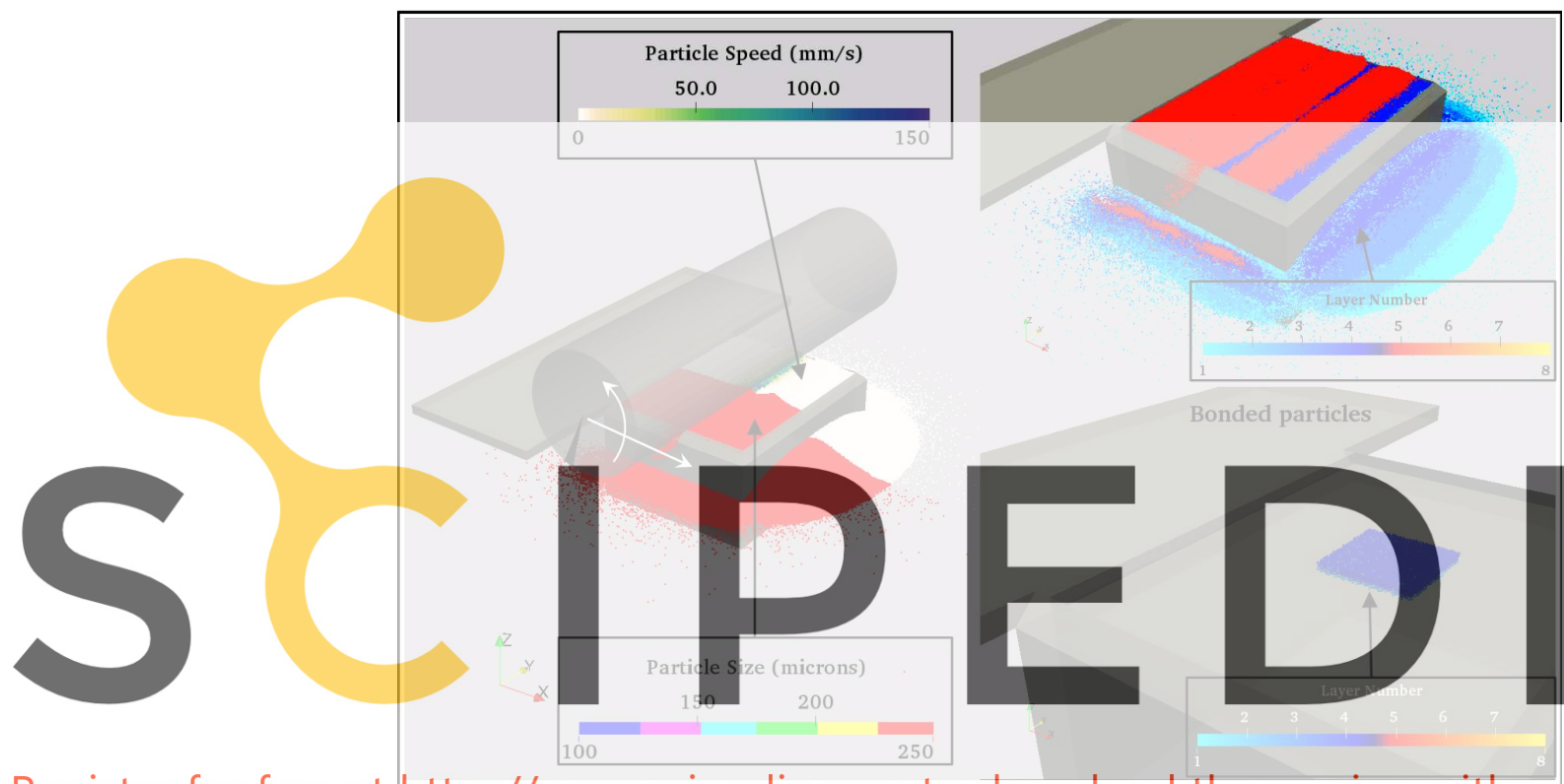

Register for free at https//www.scipedia.com to download the version without the watermark

Figure 4: Sample simulation snapshot from the multi-layer BJAM model. Left The entire simulation setup with a roller as the spreader. The roller rotates in anticlockwise direction and translates from left to right. The particles in the front-half are color-coded by their sizes and back-half are color-coded by their speeds. Upper right All powder particles color-coded by their layer numbers. Lower right Only the bonded particles are shown and are color-coded by layer numbers

\section{RESULTS}

The DEM contact parameters used in this study are listed in Table 1. A sample simulation snapshot is shown in Fig. 4. This physics-based model allows for an inspection of defects that may not lie on the current spread layer but reside buried inside the spread layers. Various particle dynamics attributes such as particle size, speed, layer number, and bond-status can be visualized using the simulation results. These attributes are color-coded in sub-images of Fig. 4. The particles simulated had a uniform size of 500 microns. However, the powder used in the BJAM experiments had an average particle size of around 50 microns. Thus the simulation results can only provide a qualitative verification and not a quantitative validation against the experimental findings. 


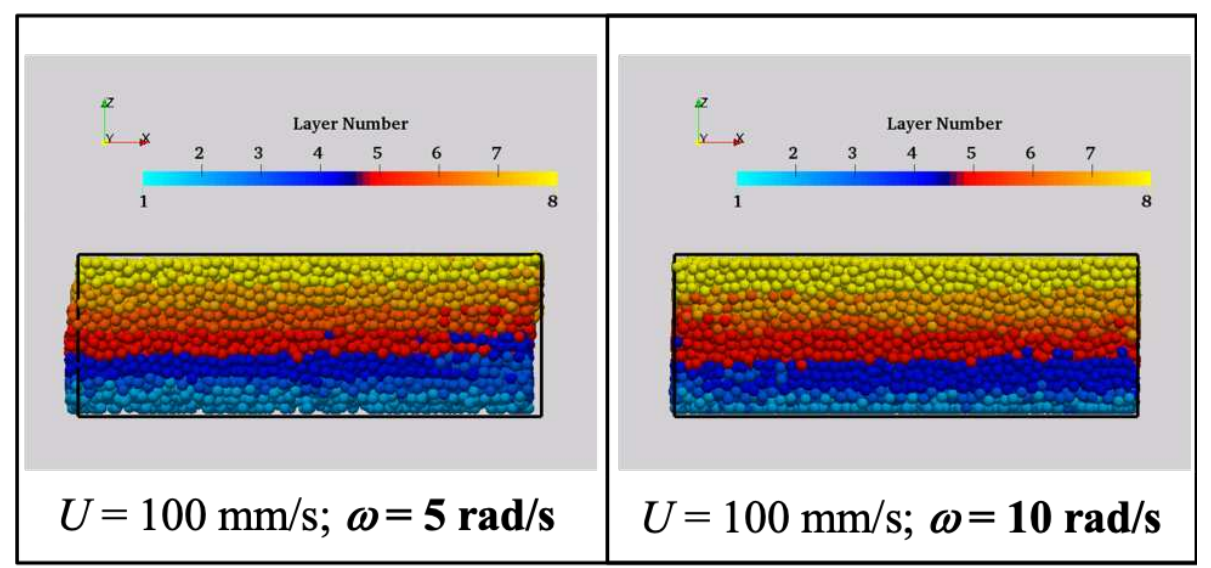

Figure 5: Virtual cuboids printed using the non-local bonded DEM model. Left Spreader shear-induced part deformation is visible at the lower layers. Shear failure of red layer (layer number 5) with respect to the dark blue layer (layer number 4) is visible along the right edge of the cuboid. Right Increasing the rotational speed of the roller fixes this shear-induced part deformation.

Cuboids with $10 \mathrm{~mm}$ by $10 \mathrm{~mm}$ by $5 \mathrm{~mm}$ in size were virtually 3D printed using the computational model at varying spreader speeds. Figure 5 shows two such virtual cuboids. The shear-induced deformation on the lower layers of the virtual cuboid in Fig. 5-left is similar to that seen in the experimental cuboid of Fig. 2 b-left. The deformation is not as prominent as the one seen in the experiments; this can be attributed to the larger particle sizes simulated when compared to the experiments. The aythors hypothesize that
simulations with correct particle sizes and distribution used in the experiments would help reduce his
disparity. However, these simulations would oe costly concerning the required computational resources.
The model correctly predicts that an increase in the spreader rotational speed to $10 \mathrm{rad} / \mathrm{s}$ would corfect the
shear-induced part deformation (1efer to Figs. 5-right and 2 b-right). The distribution of bonded farticles shear-induced part deformation (refer to Figs. 5-right and 2 b-right). The distribution of bonded particles
within layers is also quite uniform, and inter-layer mixing of bonded particles is minimized. The shear

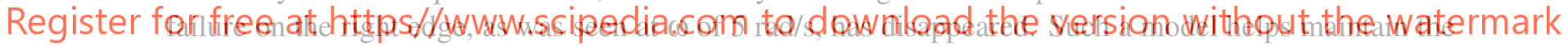
printing rate that depends on spreader translation speed and correct shear-induced defects by tweaking the rotational speed. The model can also aid newer BJAM printers that aim for pixel-wise control of properties such as color and material stiffness to minimize inter-layer mixing of bonded primitives.

\section{CONCLUSIONS}

The spreading step in binder jet additive manufacturing (BJAM) can result in various microscopic and macroscopic defects. While microscopic defects may include porosity and roughness, macroscopic defects may include under-bound and deformed parts. One class of macroscopic defects results from the shearing action of the spreader on the bound part geometry. By conducting experiments and discrete element method (DEM)modeling, the authors show the dependence of this shear-induced part deformation on spreader speeds. The binding action of the binder is modeled using a non-local bonded DEM model. The strength of bonds decays as the distance between the particle increase. The model is successfully applied to predict shear-induced deformation as a function of a spreader's rotational speed. The authors show how increasing the rotational speed and maintaining the roller's translational speed can correct the part deformation due to the spreader's shearing action. The GPU parallelized DEM model is compu- 
tationally expensive to carry out simulations at the particle sizes and counts encountered in a real 3D printer. However, the model provides initial guidance about the choice of spreader speeds before 3D printing the $\mathrm{CAD}$ file on a real $3 \mathrm{D}$ printer.

\section{ACKNOWLEDGEMENTS}

The authors thank Mr. Nicholas Wolf for his assistance in 3D printing the cuboids using an ExOne Innovent binder-jet 3D printer.

\section{REFERENCES}

[1] D. D. Gu, W. Meiners, K. Wissenbach, and R. Poprawe, "Laser additive manufacturing of metallic components: Materials, processes and mechanisms," International materials reviews, vol. 57, no. 3, pp. 133-164, 2012.

[2] K. V. Wong and A. Hernandez, "A review of additive manufacturing," International scholarly research notices, vol. 2012, 2012.

[3] W. E. Frazier, "Metal additive manufacturing: A review," Journal of Materials Engineering and Performance, vol. 23, no. 6, pp. 1917-1928, 2014.

[4] M. Li, W. Du, A. Elwany, Z. Pei, and C. Ma, "Metal binder jetting additive manufacturing: A literature review," Journal of Manufacturing Science and Engineering, vol. 142, no. 9, 2020.

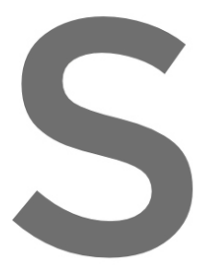

[5] M. Ziaee and N. B. Crane, "Binder jetting: A review of process, materials, and methods," Additive

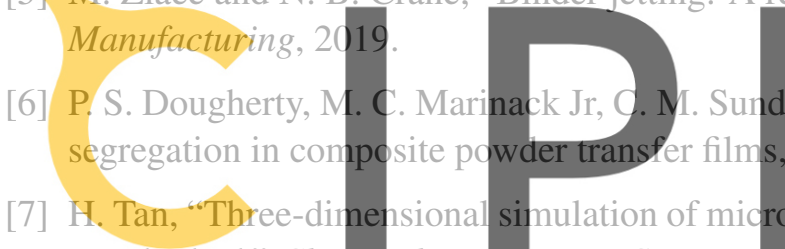
powder bed," Chemical Engineering Science, vol. 153, pp. 93-107, 2016

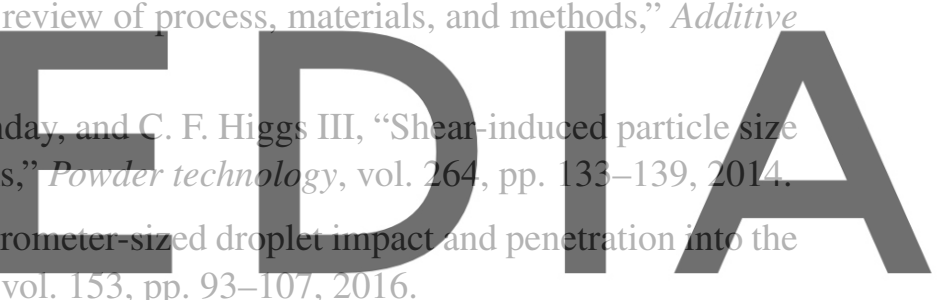

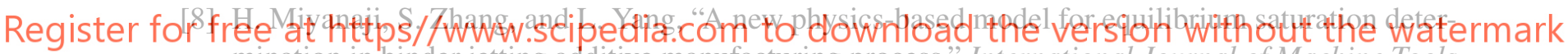
mination in binder jetting additive manufacturing process," International Journal of Machine Tools and Manufacture, vol. 124, pp. 1-11, 2018.

[9] Y. Bai, C. Wall, H. Pham, A. Esker, and C. B. Williams, "Characterizing binder-powder interaction in binder jetting additive manufacturing via sessile drop goniometry," Journal of Manufacturing Science and Engineering, vol. 141, no. 1, 2019.

[10] N. D. Parab, J. E. Barnes, C. Zhao, R. W. Cunningham, K. Fezzaa, A. D. Rollett, and T. Sun, "Real time observation of binder jetting printing process using high-speed x-ray imaging," Scientific reports, vol. 9, no. 1, pp. 1-10, 2019.

[11] P. S. Desai, A. Mehta, P. S. Dougherty, and C. F. Higgs III, "A rheometry based calibration of a first-order DEM model to generate virtual avatars of metal additive manufacturing (AM) powders," Powder Technology, vol. 342, pp. 441-456, 2019.

[12] R. Ge, M. Ghadiri, T. Bonakdar, Z. Zhou, I. Larson, and K. Hapgood, "Experimental study of the deformation and breakage of $3 \mathrm{~d}$ printed agglomerates: Effects of packing density and inter-particle bond strength," Powder technology, vol. 340, pp. 299-310, 2018. 
[13] J. Zhang, N. Amini, D. A. Morton, and K. P. Hapgood, "Binder jetting of well-controlled powder agglomerates for breakage studies," Advanced Powder Technology, 2020.

[14] S. Haeri, Y. Wang, O. Ghita, and J. Sun, "Discrete element simulation and experimental study of powder spreading process in additive manufacturing," Powder Technology, vol. 306, pp. 45-54, 2017.

[15] P. S. Desai and C. F. Higgs, "Spreading process maps for powder-bed additive manufacturing derived from physics model-based machine learning," Metals, vol. 9, no. 11, p. 1176, 2019.

[16] W. Nan and M. Ghadiri, "Numerical simulation of powder flow during spreading in additive manufacturing," Powder technology, vol. 342, pp. 801-807, 2019.

[17] H. Chen, Y. Chen, Y. Liu, Q. Wei, Y. Shi, and W. Yan, "Packing quality of powder layer during counter-rolling-type powder spreading process in additive manufacturing," International Journal of Machine Tools and Manufacture, p. 103553, 2020.

[18] H. Mindt, M. Megahed, N. Lavery, M. Holmes, and S. Brown, "Powder bed layer characteristics: the oyerseen first-order process input," Metallurgical and Materials Transactions A, vol. 47, no. 8, pp. 3811-3822, 2016.

[19] J. C. Steuben, A. P. Iliopoulos, and J. G. Michopoulos, "Discrete element modeling of particlebased additive manufacturing processes," Computer Methods in Applied Mechanics and Engineering, vol. 305, pp. 537-561, 2016.

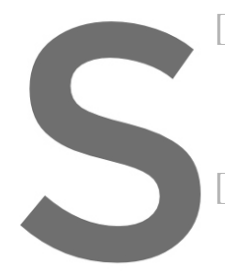

[20] L. Scime, D. Siddet, powder bed additive wise semantic segme

[21] D. Potyondy and P. mechanics and minin
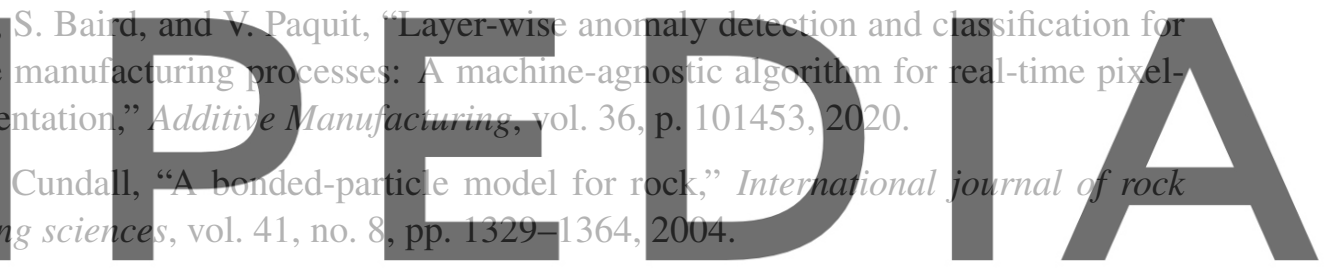

[22] P. S. Desai, "Tribosurface interactions involving particulate media with dem-calibrated properties:

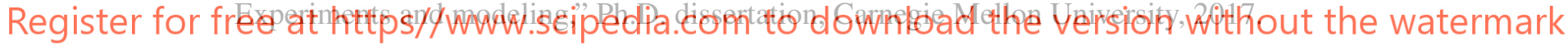

[23] D. Systèmes, SolidWorks Software, France, 2018.

[24] C. Passerello, "Interference detection using barycentric coordinates," Mechanics Research Communications, vol. 9, no. 6, pp. 373-378, 1982.

[25] L. Hu, G. Hu, Z. Fang, and Y. Zhang, "A new algorithm for contact detection between spherical particle and triangulated mesh boundary in discrete element method simulations," International journal for numerical methods in engineering, vol. 94, no. 8, pp. 787-804, 2013. 